\title{
Automated detection of patient-ventilator asynchrony: new tool or new toy?
}

\author{
Lise Piquilloud*, Philippe Jolliet and Jean-Pierre Revelly \\ See related research by Sinderby et al. http://ccforum.com/content/17/5/R239
}

\begin{abstract}
Although severe patient-ventilator asynchrony is frequent during invasive and non-invasive mechanical ventilation, diagnosing such asynchronies usually requires the presence at the bedside of an experienced clinician to assess the tracings displayed on the ventilator screen, thus explaining why evaluating patient-ventilator interaction remains a challenge in daily clinical practice. In the previous issue of Critical Care, Sinderby and colleagues present a new automated method to detect, quantify, and display patient-ventilator interaction. In this validation study, the automatic method is as efficient as experts in mechanical ventilation. This promising system could help clinicians extend their knowledge about patient-ventilator interaction and further improve assisted mechanical ventilation.
\end{abstract}

In the previous issue of Critical Care, Sinderby and colleagues [1] compare the analyses by experts and by an innovative automated method to detect and quantify patient-ventilator interaction in ventilator tracings from a previously published study. There is very good agreement between the two approaches and this opens up some exciting prospects.

Indeed, even if we have successfully used patienttriggered assisted ventilation for more than 20 years and even if (compared with controlled ventilation) this allows a reduction in sedation needs [2] and a decrease in ventilator-induced diaphragmatic dysfunction [3], we still have not solved the problem of patients 'fighting' against their ventilators. This phenomenon, commonly called patient-ventilator asynchrony [4], is related mainly to the fact that during assisted ventilation, especially during

\footnotetext{
* Correspondence: lise.piquilloud@chuv.ch

Adult Intensive Care and Burn Unit, Rue du Bugnon 46, 1011 Lausanne, Switzerland
}

pressure support, ventilator-delivered pressurization does not exactly match the characteristics of patients' inspiratory demand [5]. As a consequence, severe patientventilator asynchrony occurs in one fourth of invasively ventilated patients [6] and in more than $40 \%$ of noninvasively ventilated patients [7].

Even if patient-ventilator asynchrony is very common, studying this phenomenon remains a challenge in daily clinical practice [8]. Indeed, its correct diagnosis usually requires the presence at the bedside of an experienced clinician to assess the tracings displayed on the ventilator screen, which is not possible 24 hours a day. Additionally, up to now, a really sensitive and reliable detection of patient-ventilator asynchronies could only be performed offline by an expert using the simultaneous recording of diaphragmatic electrical activity (Eadi) flow and pressure-time curves [8], an option clearly limited to research purposes.

Only an efficient and easy-to-use automated system could help in the real-time diagnosis of patientventilator asynchronies at the bedside. As the automated system introduced by Sinderby and colleagues [1] assesses patient-ventilator interaction by automatically comparing ventilator pressure and Eadi waveforms as efficiently as experienced clinicians, it provides, for the first time, a true opportunity of continuously monitoring patient-ventilator interaction in routine clinical practice. Given that a high number of asynchronies have been associated with suboptimal ventilator settings such as excessive levels of pressure support or poorly adapted expiratory trigger setting $[9,10]$, this new monitoring tool also offers the opportunity to better adapt ventilator settings during assisted ventilation by providing realtime feedback to intensive care clinicians. Furthermore, improved closed-loop systems using the automated detection of patient-ventilator asynchronies to continuously and automatically adapt ventilator settings could be implemented in ventilators to further improve the standard of care during mechanical ventilation. 
Perhaps more importantly, poor patient-ventilator asynchrony has been associated with increased respiratory muscle workload [11], prolonged mechanical ventilation duration [6,12], and poorer outcome in difficult-to-wean patients [13]. However, whether patient-ventilator asynchronies simply occur more frequently in more severely ill patients or whether the occurrence of patient-ventilator asynchronies is by itself responsible for the poor prognosis is still unknown. Answering this important question requires large-scale clinical studies to assess the impact on patients' outcome of using ventilator strategies which can improve patient-ventilator synchrony, as, for instance, new ventilatory modes such as neurally adjusted ventilatory assist or proportional assist ventilation [14,15] or Eadi-based algorithms to adapt the ventilator settings during pressure support. However, given that, until now, analyzing patient-ventilator synchrony required manual cycleby-cycle analysis of the ventilator tracings, such largescale studies could never be performed. By allowing an automated detection, quantification, and display of patientventilator asynchronies, the system introduced by Sinderby and colleagues [1] could provide the opportunity to conduct large-scale outcome studies on the impact of correcting patient-ventilator asynchrony. Finally, this system gives the possibility of diagnosing timing errors between Eadi and pressure curves with increased sensitivity compared with standard manual analysis. This, in turn, provides an interesting new tool to further assess patientventilator synchrony and maybe to define a cutoff between acceptable and unacceptable synchrony.

In summary, the automated system presented by Sinderby and colleagues [1] to automatically detect, quantify, and display patient-ventilator asynchrony is a promising monitoring tool that should help intensive care clinicians extend their knowledge of patientventilator interaction and further improve assisted mechanical ventilation.

\section{Abbreviation}

Eadi: Diaphragmatic electrical activity.

\section{Competing interests}

The authors declare that they have no competing interests.

Published: 20 Nov 2013

\section{References}

1. Sinderby C, Liu S, Colombo D, Camarotta G, Slutsky A, Navalesi P, Beck J: An automated and standardized neural index to quantify patient-ventilator interaction. Crit Care 2013, 17:R239.

2. Girard TD, Kress JP, Fuchs BD, Thomason JW, Schweickert WD, Pun BT, Taichman DB, Dunn JG, Pohlman AS, Kinniry PA, Jackson JC, Canonico AE, Light RW, Shintani AK, Thompson JL, Gordon SM, Hall JB, Dittus RS, Bernard GR, Ely EW: Efficacy and safety of a paired sedation and ventilator weaning protocol for mechanically ventilated patients in intensive care (Awakening and Breathing Controlled trial): a randomised controlled trial. Lancet 2008, 371:126-134.
3. Jaber S, Jung B, Matecki S, Petrof BJ: Clinical review: Ventilator-induced diaphragmatic dysfunction - human studies confirm animal model findings! Crit Care 2011, 15:206.

4. Tobin MJ, Jubran A, Laghi F: Patient-ventilator interaction. Am J Respir Crit Care Med 2001, 163:1059-1063.

5. Yamada Y, Du HL: Analysis of the mechanisms of expiratory asynchrony in pressure support ventilation: a mathematical approach. J Appl Physiol 1985, 88:2143-2150.

6. Thille AW, Rodriguez P, Cabello B, Lellouche F, Brochard L: Patientventilator asynchrony during assisted mechanical ventilation. Intensive Care Med 2006, 32:1515-1522.

7. Vignaux L, Vargas F, Roeseler J, Tassaux D, Thille AW, Kossowsky MP, Brochard $L$, Jolliet P: Patient-ventilator asynchrony during non-invasive ventilation for acute respiratory failure: a multicenter study. Intensive Care Med 2009, 35:840-846.

8. Colombo D, Cammarota G, Alemani M, Carenzo L, Barra FL, Vaschetto R, Slutsky AS, Della Corte F, Navalesi P: Efficacy of ventilator waveforms observation in detecting patient-ventilator asynchrony. Crit Care Med 2011, 39:2452-2457.

9. Thille AW, Cabello B, Galia F, Lyazidi A, Brochard L: Reduction of patientventilator asynchrony by reducing tidal volume during pressure-support ventilation. Intensive Care Med 2008, 34:1477-1486.

10. Tassaux D, Gainnier M, Battisti A, Jolliet P: Impact of expiratory trigger setting on delayed cycling and inspiratory muscle workload. Am J Respir Crit Care Med 2005, 172:1283-1289.

11. Kondili E, Prinianakis G, Georgopoulos D: Patient-ventilator interaction. $\mathrm{Br} J$ Anaesth 2003, 91:106-119.

12. de Wit M, Miller KB, Green DA, Ostman HE, Gennings C, Epstein SK: Ineffective triggering predicts increased duration of mechanical ventilation. Crit Care Med 2009, 37:2740-2745.

13. Chao DC, Scheinhorn DJ, Stearn-Hassenpflug M: Patient-ventilator trigger asynchrony in prolonged mechanical ventilation. Chest 1997, 112:15921599.

14. Piquilloud L, Vignaux L, Bialais E, Roeseler J, Sottiaux T, Laterre PF, Jolliet P, Tassaux D: Neurally adjusted ventilatory assist improves patientventilator interaction. Intensive Care Med 2011, 37:263-271.

15. Alexopoulou C, Kondili E, Plataki M, Georgopoulos D: Patient-ventilator synchrony and sleep quality with proportional assist and pressure support ventilation. Intensive Care Med 2013, 39:1040-1047.

$10.1186 / \mathrm{cc} 13122$

Cite this article as: Piquilloud et al:: Automated detection of patientventilator asynchrony: new tool or new toy? Critical Care 2013, 17:1015 